\title{
Quelques caractéristiques morphométriques et de reproduction des taureaux de race Brune de l'Atlas en Algérie
}

\author{
Abdelkrim Yahimi ${ }^{1 *}$ Nadia Djellata ${ }^{1}$ Christian Hanzen ${ }^{2}$
}

\section{Mots-clés}

Bovin, taureau Brun de l'Atlas, libido, scrotum, Algérie

Submitted: 8 July 2020

Accepted: 11 March 2021

Published: 30 June 2021

DOI: $10.19182 /$ remvt.36363

\section{Résumé}

L'étude décrit les paramètres morphométriques et de reproduction, et leurs facteurs d'influence chez 249 taureaux de race Brune de l'Atlas âgés de 15,3 $\pm 3,7$ mois et pesant 165,7 $\pm 50,7 \mathrm{~kg}$ en moyenne. Les hauteurs au garrot, périmètres thoraciques et circonférences scrotales ont été respectivement de 110,8 $\pm 8,5 \mathrm{~cm}, 137,2$ $\pm 13,2 \mathrm{~cm}$ et $25,5 \pm 3,7 \mathrm{~cm}$. En ce qui concerne la libido, $12,8 \%$ des taureaux n'en ont présenté aucune et chez 22,1\% d'entre eux elle s'est avérée excellente. Les moyennes des variables étudiées en fonction de la classe d'âge ont présenté une évolution normale et progressive d'une classe à l'autre, bien que des variations très nettes aient été constatées concernant les valeurs moyennes de l'âge (AgeM), de la hauteur au garrot (HGM) et de la circonférence scrotale (CSM) à l'échelle de la ferme. Nos résultats ont également identifié des corrélations très significatives $(p<0,0001)$ entre les valeurs moyennes du tour de poitrine (TPM), de la HGM, de la CSM, et celles du poids (PoidsM) et de AgeM. De même, le modèle linaire utilisé a montré un coefficient de détermination $\left(R^{2}\right)$ très fort entre TPM, CSM, et âgeM $(0,83)$ et PoidsM $(0,62)$. En revanche, il a été très faible $\left(R^{2}=0,35\right)$ entre HGM, et AgeM et PoidsM. L'analyse de la variance des paramètres en fonction de la ferme et des classes d'âge a montré un effet très significatif $(p<0,0001)$ sur l'ensemble de l'effectif et la classe 12-18 mois. Concernant la libido, un effet significatif des scores 2 et 3 pour la classe d'âge 12-18 mois a été constaté.

- Comment citer cet article : Yahimi A., Djellata N., Hanzen C., 2021. Some morphometric and reproductive characteristics of Brown Atlas bulls in Algeria. Rev. Elev. Med. Vet. Pays Trop., 74 (2): 127134, doi: 10.19182/remvt.36363

\section{INTRODUCTION}

La population bovine locale algérienne se compose d'une seule race, la Brune de l'Atlas. Elle représente $48 \%$ du cheptel national, estimé à 300000 vaches laitières (Soukehal, 2013), mais n'assure que $20 \%$ de la production (Bencharif, 2001). Comparé à des races exotiques comme la Ndama (Coulomb, 1976), la Bourgou (Gbangboche et Alkoiret, 2011) et aux autres races de références (Angus, Simmental,

\footnotetext{
1. Institut des sciences vétérinaires, Laboratoire des biotechnologies liées à la reproduction animale, BP 270, route de Soumaa, Univ Blida 1, Algérie.

2. Université de Liège, Faculté de médecine vétérinaire, Département de gestion vétérinaire des ressources animales, 4000 Liège, Belgique.

* Auteur pour la correspondance

Email : yahimi72@yahoo.fr
}

Herford, Charolais), le bovin local est caractérisé par une production laitière très faible estimée en moyenne à 595 kilogrammes par lactation (Yakhlef et al., 2002).

La Brune de l'Atlas s'est bien adaptée au climat, au relief et aux ressources alimentaires des différentes régions du pays. Cette adaptation s'est cependant traduite par des différences de taille et de couleur de la robe en Algérie, gris foncé dans les zones forestières, blanchâtre dans les zones préforestières (figures 1 et 2). Certaines études ont caractérisé la taille (1,05 à $1,2 \mathrm{~m})$ et le poids ( 250 à $400 \mathrm{~kg}$ ) des adultes mâles et femelles (Bouzebda-Afri et al., 2007). Les capacités de reproduction des taureaux Bruns de l'Atlas ainsi que les facteurs morphométriques susceptibles de les influencer ont été très peu décrits. Pourtant, cette étape préliminaire est essentielle pour une utilisation plus rationnelle de ces taureaux car leurs capacités dépendent de l'âge, du poids, de la taille et de la circonférence scrotale (Alexander, 2008 ; Fordyce et al., 2014 ; Hanzen, 2016). 


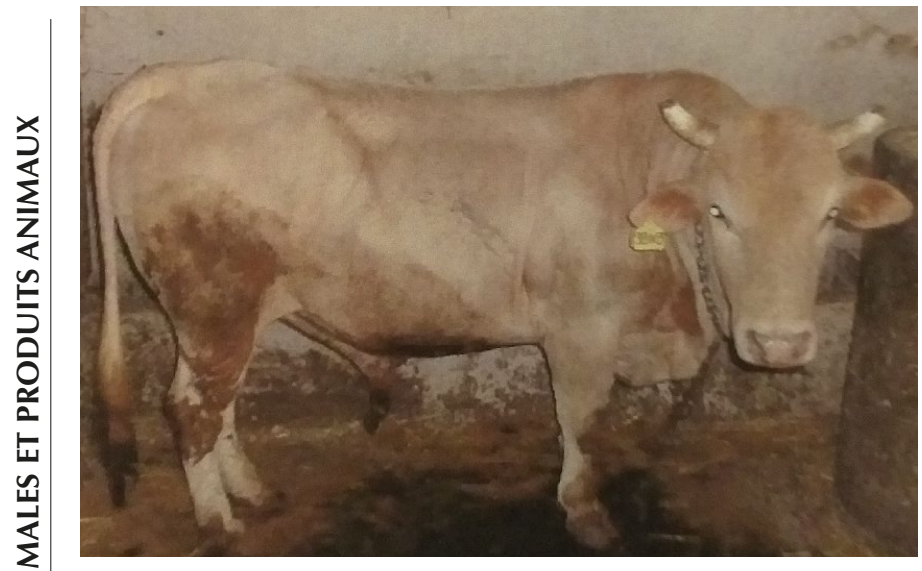

Figure 1 : Bovin de race Brune de l'Atlas à la robe gris foncé, zones forestières d'Algérie /// Brown Atlas cattle with dark gray coat, forest areas of Algeria

Classiquement, les taureaux de reproduction sont évalués sur la base de trois paramètres : la libido, la circonférence scrotale et la qualité du sperme (Alexander, 2008 ; Barth, 2018). La libido influence les caractéristiques du sperme (Ahmad et al., 2005 ; Barth, 2018) et dépend de facteurs génétiques, environnementaux (Petherick, 2005) et sociaux (Orgeur et Signoret, 1990).

La circonférence scrotale est un bon indicateur de la production quotidienne de sperme notamment chez les jeunes taureaux (McGowan et al., 2002 ; Coulter et Foote, 1979 ; Alexander, 2008). Une valeur minimale de $30 \mathrm{~cm}$ (Hopkins et Spitzer, 1997) voire de 32-33 cm chez les taureaux de grande taille (Simmental, Angus, Maine-Anjou) (Kasari et al., 1996) a été recommandée. Une étude récente (Rahmani et al., 2019) rapporte des circonférences scrotales de 13,7 et $33,5 \mathrm{~cm}$ chez des taureaux Bruns de l'Atlas respectivement âgés de 3 mois et 38-48 mois. Le présent travail a eu pour objectif de décrire la taille, le poids, la libido et la circonférence scrotale de 249 taureaux de race Brune de l'Atlas, âgés de 8 à 32 mois, et de caractériser les relations entre ces facteurs.

\section{MATERIEL ET METHODES}

\section{Données générales}

L'étude réalisée en 2016 et 2017 a concerné 249 taureaux dans 40 fermes situées dans les régions montagneuses de Bouira et Médéa, caractérisées par un climat méditerranéen chaud l'été et très froid l'hiver. Dans les fermes sélectionnées, l'élevage est de type traditionnel, avec des troupeaux de petite taille (moins de 10 têtes par élevage), et un recours assez rare aux soins et produits vétérinaires. En été, les animaux séjournent dans des pâturages naturels. En période hivernale, l'alimentation est composée d'aliments concentrés (grains de maïs, soja, son de blé et vitamines) et de foin. L'aliment est distribué deux fois par jour. Dans les troupeaux, la monte est le plus souvent libre.

L'âge des animaux a été déterminé d'après la date de naissance renseignée par l'éleveur ou, à défaut, par l'examen de la dentition de l'animal. Le poids corporel (PV) a été estimé à partir de la mesure du périmètre thoracique (PT) en utilisant la formule de Crevat : $\left(P V=80 \times P T^{3}\right)($ Marmet, 1983). Le périmètre thoracique a été mesuré avec un ruban métrique placé en arrière des épaules et la hauteur au garrot avec une toise.

La circonférence scrotale a été déterminée de la manière suivante (Hanzen, 2016) : « une fois le contenu scrotal palpé, les testicules sont positionnés fermement au fond des bourses testiculaires en appliquant

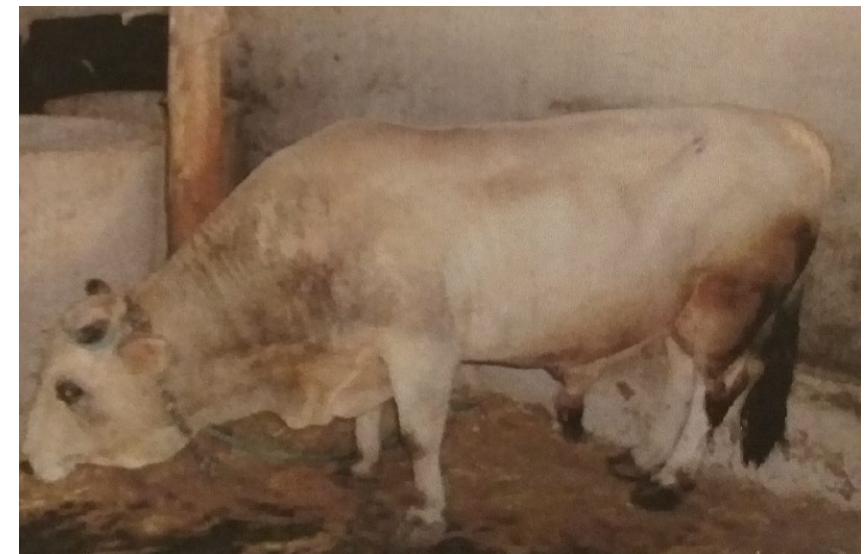

Figure 2 : Bovin de race Brune de l'Atlas à la robe blanchâtre, zones préforestières d'Algérie /// Brown Atlas cattle with a whitish coat, preforest areas of Algeria

une main au niveau des cordons testiculaires en évitant de placer un doigt entre les testicules. La pression ainsi exercée ne doit pas être excessive pour éviter un écartement anormal des testicules. Un mètre ruban est ensuite placé autour du plus grand diamètre des testicules et serré de manière telle qu'il assure un simple contact avec le scrotum ». Cette technique a été réalisée après une bonne contention de l'animal.

« La libido consiste en la réaction de l'animal en présence d'une femelle en chaleurs. On déterminera le nombre de montes réalisées pendant une période de 15 à 20 minutes. Si au bout de cette période, aucune voire une seule monte a été effectuée, on peut estimer la libido comme faible. Elle est considérée comme moyenne si 2 à 3 montes ont été réalisées et comme excellente si ce nombre est égal ou supérieur à 4 » (Hanzen, 2016).

\section{Analyses statistiques}

Les données récoltées ont été analysées avec le logiciel XLSTAT (vers. 2016.02.28451). Les variables quantitatives, moyennes, écartstypes et valeurs extrêmes ont été calculées pour les 249 taureaux, ainsi que pour les classes d'âge 8-11 mois, 12-18 mois et 24-30 mois. Une analyse de la variance a été appliquée pour déterminer l'effet de l'âge moyen (AgeM) et du poids moyen (PoidsM) sur l'évolution de la valeur moyenne des variables suivantes : hauteur au garrot (HGM), tour de poitrine (TPM) et circonférence scrotale (CSM) à l'échelle de la ferme. Ensuite, une analyse de variance a été réalisée pour déterminer la relation entre l'évolution de la circonférence scrotale (variable à expliquer) en fonction de l'âge, du tour de poitrine, de la hauteur au garrot, du poids vif des animaux, de la libido et de la ferme (variables explicatives), suivie d'une analyse de la relation de l'âge et du poids moyen avec la hauteur au garrot, le tour de poitrine et la circonférence scrotale par ferme. Afin d'étudier l'effet ferme et la relation entre libido et taille de la circonférence scrotale, plusieurs tests de comparaisons multiples ont été réalisés : test de Tukey, méthode REGWQ et test de Dunnet. Le seuil d'erreur retenu a été de $5 \%$.

\section{RESULTATS}

Les résultats descriptifs des paramètres morphométriques des 249 taureaux âgés de 15,4 $\pm 3,9$ mois ont révélé des valeurs moyennes du poids, de la circonférence scrotale, de la hauteur au garrot et du périmètre thoracique, respectivement de $165,7 \pm 50,7 \mathrm{~kg}, 25,5 \pm 3,7 \mathrm{~cm}$, $110,8 \pm 8,5 \mathrm{~cm}$ et $136,9 \pm 13,1 \mathrm{~cm}$ (tableau I). L'évaluation de la libido a montré une majorité $(65,5 \%)$ de scores moyens. La classe d'âge 
24-30 mois a montré une ardeur sexuelle très élevée $(67,8 \%)$ par rapport aux autres classes d'âge.

Pour ce qui est de l'évolution de la HGM, de la CSM et de l'âge au niveau de chaque ferme, des variations marquées ont été constatées (figures $3,4,5$ ) notamment au niveau des fermes 28,29 et 7 où l'âge moyen était supérieur à 19 mois, avec respectivement des HGM de $115,9,106$ et $115,4 \mathrm{~cm}$, et des CSM de 24, 23 et $25 \mathrm{~cm}$.

En revanche, pour un AgeM de 16,6 mois dans les fermes 1 et 27, une HGM de plus de $119 \mathrm{~cm}$ a été enregistrée. D'une manière générale, les valeurs moyennes des différents paramètres par ferme ont été plus

Tableau I : Paramètres morphométriques et libido selon la classe d'âge des taureaux Bruns de l'Atlas en Algérie /// Morphometric parameters and libido according to age class of Brown Atlas bulls in Algeria

\begin{tabular}{|c|c|c|c|c|c|c|c|c|c|c|c|c|}
\hline \multirow[t]{2}{*}{ Variable } & \multicolumn{3}{|c|}{ Effectif total $(n=249)$} & \multicolumn{3}{|c|}{$8-11$ mois $(n=16)$} & \multicolumn{3}{|c|}{$12-18$ mois $(n=205)$} & \multicolumn{3}{|c|}{$24-30$ mois $(n=28)$} \\
\hline & Moy ET & Min & Max & Moy \pm ET & Min & $\operatorname{Max}$ & Moy \pm ET & Min & Max & Moy \pm ET & Min & Max \\
\hline $\mathrm{CS}(\mathrm{cm})$ & $25,49 \pm 3,7$ & 14,5 & 35,0 & $18,5 \pm 3,3$ & 14,5 & 22,0 & $25,4 \pm 2,7$ & 15,5 & 32,0 & $29,9 \pm 3,7$ & 21,5 & 35,0 \\
\hline Age (mois) & $15,29 \pm 3,8$ & 8,0 & 30,0 & $9,3 \pm 1,3$ & 8,0 & 11,0 & $14,5 \pm 1,7$ & 12,0 & 18,0 & $24,1 \pm 1,3$ & 23,0 & 30,0 \\
\hline $\mathrm{TP}(\mathrm{cm})$ & $136,9 \pm 13,1$ & 109,0 & 172,0 & $123,2 \pm 11,9$ & 109,0 & 142,0 & $136,6 \pm 11,6$ & 117,0 & 172,0 & $149,6 \pm 13,8$ & 126,0 & 172,0 \\
\hline $\mathrm{HG}(\mathrm{cm})$ & $110,8 \pm 8,5$ & 90,0 & 135,0 & $101,5 \pm 6,3$ & 90,0 & 107,0 & $111,1 \pm 8,2$ & 96,0 & 135,0 & $114,2 \pm 8,3$ & 99,5 & 127,0 \\
\hline Poids (kg) & $165,7 \pm 50,7$ & 75,0 & 300,0 & $92,8 \pm 14,0$ & 75,0 & 120,0 & $164,1 \pm 45,4$ & 75,0 & 300,0 & $219,2 \pm 43,1$ & 140,0 & 275,0 \\
\hline \multirow[t]{4}{*}{ Libido } & Score & $\mathbf{N}$ & $\%$ & Score & $\mathbf{N}$ & $\%$ & Score & $\mathbf{N}$ & $\%$ & Score & $\mathbf{N}$ & $\%$ \\
\hline & 1 & 32 & 12,8 & 1 & 11 & 68,8 & 1 & 17 & 8,3 & 1 & 4 & 14,3 \\
\hline & 2 & 162 & 65,5 & 2 & 5 & 31,2 & 2 & 152 & 74,1 & 2 & 5 & 17,9 \\
\hline & 3 & 55 & 22,1 & 3 & - & - & 3 & 36 & 17,6 & 3 & 19 & 67,8 \\
\hline
\end{tabular}

Moy \pm ET : moyenne \pm écart-type $;$ Max $:$ maximum $;$ Min : minimum $;$ CS : circonférence scrotale $;$ TP : tour de poitrine $;$ HG : hauteur au garrot ; Score $1=$ faible, $2=$ moyen, $3=$ excellent /// Moy \pm ET: mean \pm standard deviation; Max: maximum; Min: minimum; CS: scrotal circumference; TP: chest circumference; HG: height at withers; Score 1 = poor, 2 = average, $3=$ excellent

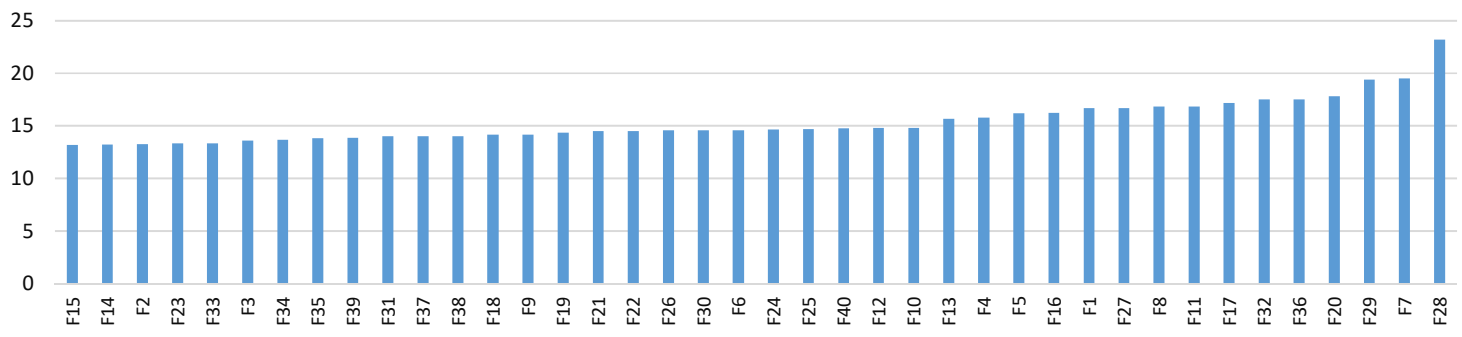

Figure 3 : Age moyen (mois) des taureaux Bruns de l'Atlas dans 40 fermes algériennes /// Mean age (months) of Brown Atlas bulls in 40 Algerian farms

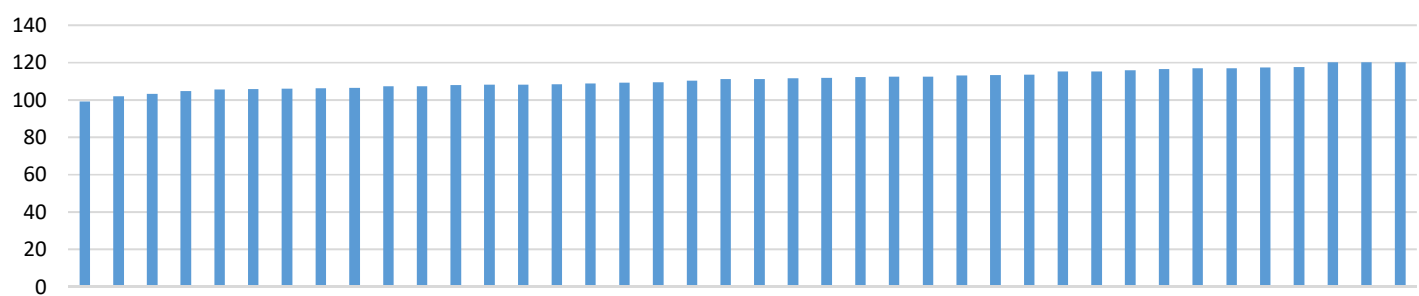

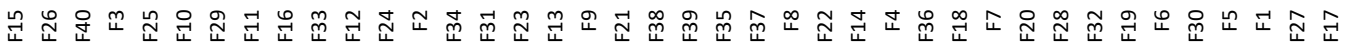

Figure 4 : Hauteur au garrot moyenne $(\mathrm{cm})$ des taureaux Bruns de l'Atlas dans 40 fermes algériennes /// Mean height at withers $(\mathrm{cm})$ of Brown Atlas bulls in 40 Algerian farms

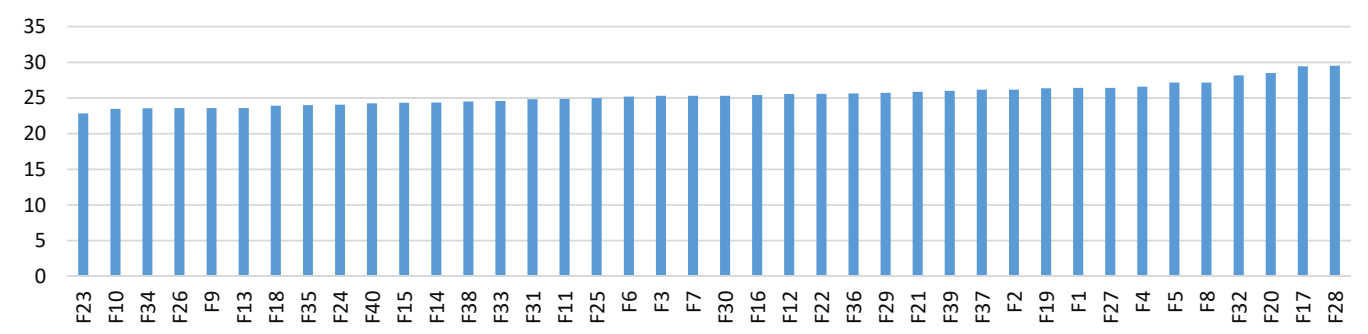

Figure 5 : Circonférence scrotale moyenne $(\mathrm{cm})$ des taureaux Bruns de l'Atlas dans 40 fermes algériennes /// Mean scrotal circumference (cm) of Brown Atlas bulls in 40 Algerian farms 
ou moins proches. Concernant la relation entre la libido et la CSM, celle-ci était égale à $23,4 \mathrm{~cm}, 25,6 \mathrm{~cm}$ et $26,3 \mathrm{~cm}$ respectivement pour les taureaux dont la libido était faible (score 1), moyenne (score 2) et excellente (score 3).

Concernant les interactions entre les mensurations (TPM, HGM, CSM), et AgeM et PoidsM par ferme, le modèle linéaire généralisé a mis en évidence un effet principal ( $p<0,001)$ (tableau II) de ces mensurations, des interactions entre TPM, HGM et PoidsM ( $p<0,001)$, et entre AgeM, CSM et PoidsM ( $p<0,5)$. En revanche, nous n'avons pas observé d'interaction entre HGM et AgeM par ferme $(\mathrm{p}=0,61)$.

L'analyse de la variance sur l'effectif global (249) et pour les groupes 8-11 mois, 12-18 mois et 24-30 mois a donné des coefficients de détermination multiple $\left(R^{2}\right)$ respectivement de $0,75,0,51,0,71$ et 0,85 (tableau III). Le coefficient de détermination plus proche de 1 révèle l'existence d'une plus forte variabilité entre les sujets étudiés. Les analyses de la variance sur l'effectif total (249) et sur la classe d'âge (12-18 mois) ont montré une relation très significative $(\mathrm{p}<0,0001)$ entre l'évolution de la circonférence scrotale selon l'âge, le poids vif, et les scores 2 et 3 de la libido (tableau IV). En revanche, aucun effet n'a été observé pour les classes d'âge 8-11 mois et 24-30 mois.

S'agissant des variations de la moyenne de la circonférence scrotale en fonction des fermes, le test de Tukey HSD (honestly significantly different) réalisé par couple de fermes n'a pas permis de relever de différences entre les fermes. Les mêmes conclusions ont été constatées avec la méthode REGWQ et le test de Dunnet bilatéral. Concernant la relation entre la libido et la circonférence scrotale, une différence significative $(\mathrm{p}<0,05)$ pour les taureaux présentant un score de 2 et 3 a été observée, notamment pour la classe (12-18 mois) (tableau IV), mais pas pour le score 1 .

Tableau II : Interactions des paramètres morphométriques avec l'âge et le poids moyens par ferme chez des taureaux Bruns de l'Atlas en Algérie (régression linéaire) /// Interactions of morphometric parameters with the mean age and weight by farm in Brown Atlas bulls in Algeria (linear regression)

\begin{tabular}{|c|c|c|c|c|c|c|c|}
\hline \multicolumn{2}{|l|}{ Variable } & \multirow{2}{*}{$\begin{array}{c}\text { DDL } \\
1\end{array}$} & \multirow{2}{*}{$\begin{array}{c}\text { REP } \\
93,126\end{array}$} & \multirow{2}{*}{$\begin{array}{c}\mathbf{t} \\
24,62\end{array}$} & \multirow{2}{*}{$\begin{array}{c}\mathbf{P} \\
<0,0001\end{array}$} & \multirow{2}{*}{$\begin{array}{l}\text { Equation } \\
\text { TPM }=93,12+0,77 \text { AgeM+0,19PoidsM }\end{array}$} & \multirow{2}{*}{$\begin{array}{c}\mathbf{R}^{\mathbf{2}} \\
0,83\end{array}$} \\
\hline TPM & Interaction & & & & & & \\
\hline & AgeM & 1 & 0,779 & 2,27 & 0,0290 & & \\
\hline & PoidsM & 1 & 0,19 & 8,13 & $<0,0001$ & & \\
\hline \multirow[t]{3}{*}{ HGM } & Interaction & 1 & 95,056 & 19,16 & $<0,0001$ & HGM $=95,05-0,22$ AgeM+0,11 PoidsM & 0,35 \\
\hline & AgeM & 1 & $-0,226$ & $-0,50$ & 0,6175 & & \\
\hline & PoidsM & 1 & 0,116 & 3,75 & 0,0006 & & \\
\hline \multirow[t]{3}{*}{ CSM } & Interaction & 1 & 16,421 & 12,95 & $<0,0001$ & CSM $=16,44+0,30$ Age $M+0,02$ PoidsM & 0,62 \\
\hline & AgeM & 1 & 0,305 & 2,66 & 0,0114 & & \\
\hline & PoidsM & 1 & 0,026 & 3,33 & 0,002 & & \\
\hline
\end{tabular}

DDL : degré de liberté ; REP : résultats estimés des paramètres ; $t$ : test de Student ; TPM : moyenne du tour de poitrine ; HGM : moyenne de la hauteur au garrot ; CSM : moyenne de la circonférence scrotale //I DDL: degree of freedom; REP: estimated results of the parameters; $t$ : Student t test; TPM: mean chest circumference; HGM: mean height at withers; CSM: mean scrotal circumference

Tableau III : Evolution de la circonférence scrotale de l'effectif total de taureaux Bruns de l'Atlas en fonction de la classe d'âge en Algérie III Evolution of the scrotal circumference of the total number of Brown Atlas bulls according to age class in Algeria

\begin{tabular}{|c|c|c|c|c|c|c|}
\hline & DDL & SdC & MdC & $\mathbf{R}^{2}$ & $\mathbf{F}$ & $P>F$ \\
\hline \multicolumn{7}{|c|}{ Effectif total (249) } \\
\hline Modèle & 45 & 2582,53 & 57,39 & 0,753 & 13,72 & $<0,0001$ \\
\hline Erreur & 203 & 848,85 & 4,182 & & & \\
\hline Total corrigé & 248 & 3431,38 & & & & \\
\hline \multicolumn{7}{|c|}{ Classe d'âge $8-11$ mois $(n=16)$} \\
\hline Modèle & 5 & 88,34 & 17,66 & 0,515 & 2,12 & 0,145 \\
\hline Erreur & 10 & 83,09 & 8,30 & & & \\
\hline Total corrigé & 15 & 171,43 & & & & \\
\hline \multicolumn{7}{|c|}{ Classe d'âge 12-18 mois $(n=205)$} \\
\hline Modèle & 45 & 1125,40 & 25,00 & 0,715 & 8,85 & $<0,0001$ \\
\hline Erreur & 159 & 448,84 & 2,82 & & & \\
\hline Total corrigé & 204 & 1574,24 & & & & \\
\hline \multicolumn{7}{|c|}{ Classe d'âge $24-30$ mois $(n=28)$} \\
\hline Modèle & 21 & 314,67 & 14,98 & 0,848 & 1,59 & 0,292 \\
\hline Erreur & 6 & 56,25 & 9,37 & & & \\
\hline Total corrigé & 27 & 370,93 & & & & \\
\hline
\end{tabular}

DDL : degré de liberté ; SdC : somme des carrés ; MdC : moyennes des carrés ; $\mathrm{R}^{2}$ : coefficient de détermination ; F : test de Fisher /// DDL: degree of freedom; SdC: sum of squares; MdC: mean of squares; $R^{2}$ : coefficient of determination; F: Fisher test 
Tableau IV : Relation de la circonférence scrotale des taureaux Bruns de l'Atlas avec des paramètres morphométriques et la libido en fonction de l'âge et de la ferme en Algérie //I Relationship of the scrotal circumference of Brown Atlas bulls with morphometric parameters and libido in relation to age and farm in Algeria

\begin{tabular}{|c|c|c|c|c|c|c|}
\hline & Valeur & ES & $\mathbf{t}$ & $\mathbf{P}>|\mathbf{t}|$ & BI $(95 \%)$ & BS $(95 \%)$ \\
\hline \multicolumn{7}{|c|}{ Effectif total $(n=249)$} \\
\hline Constante & 14,021 & 2,865 & 4,894 & $<0,0001$ & 8,372 & 19,670 \\
\hline Age & 0,236 & 0,055 & 4,305 & $<0,0001$ & 0,128 & 0,345 \\
\hline TP & $-0,009$ & 0,020 & $-0,475$ & 0,635 & $-0,048$ & 0,029 \\
\hline HG & $-0,008$ & 0,023 & $-0,363$ & 0,717 & $-0,053$ & 0,037 \\
\hline Poids & 0,044 & 0,006 & 7,968 & $<0,0001$ & 0,033 & 0,055 \\
\hline Libido-1 & 0,000 & 0,000 & & - & - & - \\
\hline Libido-2 & 2,199 & 0,449 & 4,898 & $<0,0001$ & 1,314 & 3,084 \\
\hline Libido-3 & 2,877 & 0,630 & 4,565 & $<0,0001$ & 1,634 & 4,120 \\
\hline \multicolumn{7}{|c|}{ Classe d'âge 8-11 mois $(n=16)$} \\
\hline Constante & 213,640 & 89,393 & 2,390 & 0,038 & 14,459 & 412,820 \\
\hline Age & 0,824 & 1,127 & 0,731 & 0,482 & $-1,687$ & 3,334 \\
\hline TP & 0,821 & 0,401 & 2,047 & 0,068 & $-0,073$ & 1,715 \\
\hline $\mathrm{HG}$ & $-2,320$ & 1,044 & $-2,223$ & 0,050 & $-4,646$ & 0,005 \\
\hline Poids & $-0,655$ & 0,311 & $-2,107$ & 0,061 & $-1,347$ & 0,038 \\
\hline Libido-1 & 0,000 & 0,000 & & & & \\
\hline Libido-2 & $-24,712$ & 12,458 & $-1,984$ & 0,075 & $-52,469$ & 3,045 \\
\hline \multicolumn{7}{|c|}{ Classe d'âge $12-18$ mois $(n=205)$} \\
\hline Constante & 15,615 & 2,812 & 5,552 & $<0,0001$ & 10,061 & 21,170 \\
\hline Age & $-0,053$ & 0,111 & $-0,473$ & 0,637 & $-0,272$ & 0,167 \\
\hline TP & 0,031 & 0,019 & 1,657 & 0,100 & $-0,006$ & 0,069 \\
\hline HG & $-0,031$ & & $-1,485$ & 0,140 & $-0,072$ & 0,010 \\
\hline Poids & 0,040 & & 7,402 & $<0,0001$ & 0,029 & 0,050 \\
\hline Libido-1 & 0,000 & & & & & \\
\hline Libido-2 & 1,870 & 0,480 & 3,899 & 0,000 & 0,923 & 2,817 \\
\hline Libido-3 & 2,350 & 0,658 & 3,572 & 0,000 & 1,050 & 3,649 \\
\hline \multicolumn{7}{|c|}{ Classe d'âge $24-30$ mois $(n=28)$} \\
\hline Constante & 16,327 & 38,210 & 0,427 & 0,684 & $-77,169$ & 109,823 \\
\hline Age & 0,911 & 1,296 & 0,703 & 0,508 & $-2,259$ & 4,082 \\
\hline TP & 0,029 & 0,208 & 0,139 & 0,894 & $-0,480$ & 0,538 \\
\hline HG & $-0,104$ & 0,021 & $-0,252$ & 0,810 & $-1,111$ & 0,904 \\
\hline Poids & 0,002 & 0,005 & 0,021 & 0,984 & $-0,236$ & 0,240 \\
\hline Libido-1 & 0,000 & 0,000 & & & & \\
\hline Libido-2 & $-2,763$ & 6,915 & $-0,400$ & 0,703 & $-19,683$ & 14,157 \\
\hline Libido-3 & 2,131 & 4,437 & 0,480 & 0,648 & $-8,725$ & 12,987 \\
\hline
\end{tabular}

$\mathrm{TP}$ : tour poitrine ; HG : hauteur au garrot ; ES : erreur standard ; $\mathrm{t}$ : test t de Student ; BI : borne inférieure ; BS : borne supérieure /// TP: chest circumference; HG: height at withers; ES: standard error; $t$ : Student t test; BI: lower limit; BS: upper limit

\section{DISCUSSION}

Les données morphométriques (hauteur au garrot, poids, âge, tour de poitrine) et de reproduction (circonférence scrotale, qualité de la libido) ont permis de relever de nettes variations d'un animal à l'autre. Les taureaux Bruns de l'Atlas font partie des taureaux de petite taille. Leur taille moyenne $(110,9 \pm 8,6 \mathrm{~cm})$ enregistrée à un âge moyen de $15,4 \pm 3,9$ mois est caractéristique des races locales bien adaptées à des conditions et un environnement particuliers (Couix et al., 2016). Elle est très nettement inférieure aux tailles des taureaux adultes Angus (144 cm) ou Limousins (137 cm, Bene et al., 2007). Les différences de conformation et de taille des races sont liées généralement à la valeur génétique et au type d'alimentation distribué (FAO, 2013).
Dans notre suivi, les moyennes des paramètres étudiés par classe d'âge ont montré une évolution cohérente où la hauteur au garrot, le tour de poitrine, la circonférence scrotale et le poids ont suivi un développement normal lié à l'âge. L'accroissement de ces paramètres a été observé de façon plus nette chez les jeunes que chez les adultes suivant ainsi l'allure curvilinéaire de la courbe de croissance (Delage et al., 1955). Leurs valeurs restent cependant éloignées de celles des taureaux d'autres races standards. Ces différences s'expliquent par le caractère spécifique de cette race, sa rusticité et son adaptation au milieu et aux conditions climatiques, qui fondent son originalité.

Les valeurs obtenues étaient cependant inférieures à celles observées en Algérie chez 196 taureaux Bruns de l'Atlas, âgés de 12 à 48 mois (121 cm, Rahmani et al. 2020) ou sur des taureaux Ndama au 
Congo-Brazzaville (115 cm, Akouango et al., 2010). Agés de 22 mois, leur poids moyen $(212 \mathrm{~kg})$ s'est avéré inférieur à celui observé chez les taureaux Borgou au Bénin (300 kg) âgés de 36 mois (Dehoux et Hounsou, 1993). Ces deux caractères, notamment la hauteur au garrot et le poids, sont des indicateurs indirects de production et des conditions d'élevage (FAO, 2013).

Concernant le périmètre thoracique, une valeur moyenne de 136,9 $\pm 13,1 \mathrm{~cm}$ a été constatée. Cette valeur a varié de $123,2 \mathrm{~cm}$ à $8-11$ mois à 149,6 cm à 24-30 mois, montrant un développement très distinctif avec une différence de plus de $20 \mathrm{~cm}$, suivant les lois générales de la croissance (Coulomb, 1976). La valeur du périmètre thoracique dans notre étude a été inférieure à celles observées en Algérie chez des taureaux Bruns de l'Atlas âgés de plus de 24 mois $(155 \mathrm{~cm}$, Rahmani et al., 2020) et Ndama âgés de plus de 36 mois $(158 \mathrm{~cm}$, Akouango et al., 2014). Les âges moyens différents mais aussi les conditions d'élevage et d'alimentation ne sont sans doute pas étrangers aux différences observées. D'une manière générale, tous les paramètres (tour de poitrine, hauteur au garrot, poids et circonférence scrotale) ont enregistré une évolution progressive en fonction de l'âge, témoignant de l'absence de rupture dans les apports alimentaires selon les saisons.

Concernant les variations des valeurs moyennes de la hauteur au garrot, l'âge et la circonférence scrotale, les représentations graphiques ont montré des différences plus ou moins nettes d'une ferme à l'autre. Une HGM de $120 \mathrm{~cm}$ et une CSM de plus de $26 \mathrm{~cm}$ pour un âge moyen de plus de 15 mois dans la ferme 27 ont été constatées, alors que dans la ferme 1, la HGM était de moins de $120 \mathrm{~cm}$ et la CSM de moins de $25 \mathrm{~cm}$ pour un âge moyen de plus de 20 mois. Ces variations sont généralement dépendantes des conditions d'élevage, de la génétique et des objectifs tracés par les éleveurs.

L'augmentation de la circonférence scrotale était particulièrement notable entre la première et la deuxième classe d'âge (plus $6,5 \mathrm{~cm}$ ), ce qui s'expliquait par l'accélération du développement testiculaire essentiel de l'évaluation de la capacité de reproduction des taureaux (Chenoweth, 2010). Elle est corrélée au volume du parenchyme testiculaire et donc à la production de spermatozoïdes de qualité. Notre valeur observée était comparable à celles rapportées pour des taureaux de taille semblable : $22,7 \mathrm{~cm}$ a été observée chez des taureaux de race Ndama d'un poids moyen de $151 \mathrm{~kg}$ (Akouango et al., 2010). Des valeurs de $29,75 \pm 3,18 \mathrm{~cm}, 27,5 \pm 2,12 \mathrm{~cm}$ et $28,02 \pm 2,23 \mathrm{~cm}$ ont été mentionnées respectivement pour des taureaux Bos indicus de race Borgou, Azawak (Akpo et al., 2018) et Aceh (Dasrul et al., 2020). Une valeur moyenne de $25,9 \mathrm{~cm}$ a été observée chez des taureaux de race Brune de l'Atlas âgés de 12-18 mois par Rahmani et al. (2019). Ces différentes circonférences scrotales sont en revanche bien inférieures à celles de taureaux Brangus et Nelore âgés de 12 à 36 mois (respectivement 37,6 et 35,2 cm) (Hartmann et al., 2018). La circonférence scrotale dépendait de l'âge mais également du poids de l'animal. De semblables corrélations ont également été observées chez différentes races de taureaux en Australie (Fordyce et al., 2014). La circonférence scrotale dépend également de la race mais aussi de son alimentation à un âge et dans un environnement donné (Schramm et al., 1989 ; Parkinson, 2004 ; Akouango et al., 2010 ; Fordyce et al., 2014). Ainsi, en Australie, une circonférence minimale de 34 à $35 \mathrm{~cm}$ a été recommandée pour différentes races de Bos taurus (Fordyce et al., 2014). Selon Chenoweth et al. (2010), les valeurs de la relation entre la circonférence scrotale et l'âge devraient être idéalement supérieures à $34 \mathrm{~cm}$ et $39 \mathrm{~cm}$, respectivement à 15 et 24 mois. Une valeur moyenne minimale de $30 \mathrm{~cm}$ a été recommandée au Canada pour des taureaux âgés de 12-30 mois ; cette valeur pouvant être différente selon la race (Coulter et al., 1987). Selon Fordyce et al. (2014), des taureaux de races tempérées élevés en milieu tropical peuvent présenter une circonférence inférieure à celle de taureaux de même race élevés en milieu tempéré. Par ailleurs, ces auteurs insistent sur le fait qu'une circonférence inférieure à $20 \mathrm{~cm}$ est probablement due à une mesure erronée. Une prédiction acceptable de la circonférence scrotale à partir du poids a été observée lorsque le poids des taureaux était compris entre 250 et $750 \mathrm{~kg}$. L'alimentation exerce un effet dès la période prépubertaire (Barth et al., 2008 ; Kastelic et Thundathil, 2008).

Lévaluation de la libido constitue également un important paramètre d'évaluation de la capacité de reproduction d'un taureau. Plus de $12 \%$ des taureaux étudiés présentaient un manque de libido. Un problème semblable a été rapporté chez la Ndama (Moupandza et al., 2013). Une mauvaise gestion de l'élevage (suivi sanitaire, alimentation) influence fortement le développement normal des paramètres de reproduction ou morphométriques dans les fermes de cette race. La libido dépendrait aussi de la génétique, de l'alimentation, des conditions climatiques et de l'état de santé de l'animal. Par ailleurs, les taureaux de race laitière présenteraient une libido plus précoce et plus importante que les taureaux de race à viande (Dumont, 1997). Plus elle est manifestée et meilleure serait la fertilité (Birkner et al., 1984).

\section{CONCLUSION}

Cette étude a permis de préciser les caractéristiques morphométriques et de reproduction des taureaux de la race locale algérienne Brune de l'Atlas. Malgré des conditions d'élevage difficiles, les taureaux ont présenté des caractères morphologiques acceptables ainsi que des capacités de reproduction qui se traduisaient par un temps de monte rapide, une bonne libido et une circonférence scrotale satisfaisante. Ils présentaient néanmoins des différences par rapport aux standards des races bovines, comparables en terme de gabarit et le plus souvent étudiées.

Les données décrites dans la présente étude constituent une première étape à l'élaboration d'une base de données nationale qui devrait permettre de mieux appréhender les facteurs génétiques, alimentaires ou environnementaux susceptibles d'en être responsables. La prise en compte systématique de ces résultats par les responsables de santé permettrait de mieux identifier les animaux aptes à la reproduction.

\section{Déclaration des contributions des auteurs}

AY a participé à la conception et à la planification de l'étude, a récolté et analysé les données, et a participé à la rédaction du manuscrit ; ND a participé à la révision critique du document ; $\mathrm{CH}$ a participé à la conception et à la planification de l'étude, à l'interprétation des résultats et à la rédaction de la première version.

\section{Conflits d'intérêts}

Leétude a été réalisée sans conflit d'intérêts.

\section{REFERENCES}

Ahmad M., Asmat M., Rehman N., 2005. Relationship of testicular size and libido to age and season in Sahiwal bulls. Pakistan Vet. J., 25 (2): 67

Akouango F., Ngokaka C., Ewomango P., Kimbembe E., 2010. Caractérisation morphométrique et reproductive des taureaux et vaches N'Dama du Congo. Anim. Gen. Res., 4: 41-47, doi: 10.1017/S2078633610000688

Akouango P., Mopoundza P., Ewomango R., 2014. Etude des mensurations des bovins de race Ndama (Bos taurus) dans les pâturages naturels semi inondés de la ferme d'Abo au Congo-Brazzaville. J. Anim. Plant Sci., 20 (3): 3137-3143

Akpo Y., Mehouenou C.G.L., Yessinou R.E., Alkoiret Traore I., Kpodekon M.T., 2018. Evaluation de la qualité des semences issues des taureaux de races Borgou, Azawak et Girolando utilisés au Centre national d'insémination artificielle bovine au Bénin. Ann. Univ. Parakou, 8: 13-21

Alexander J.H., 2008. Bull breeding soundness evaluation: A practitioner's perspective. Theriogenology, 70: 469-472, doi: 10.1016/j.theriogenology.2008.05.030 
Barth A.D., 2018. The use of bull breeding soundness evaluation to identify sub fertile and infertile bulls. Animal, 12 (s1): s158-s164, doi: 10.1017/ S1751731118000538

Barth A.D., Ominski K.H., 2000. The relationship between scrotal circumference at weaning and at one y of age in the beef bull. Can. Vet. J., 40: 541546

Barth A.D, Brito L.F.C, Kastelic J.P., 2008. The effect of nutrition on sexual Development of bulls. Theriogenology, 70: 485-94, doi: 10.1016/j.theriogenology.2008.05.031

Bencharif A., 2001. Stratégies des acteurs de le filières lait en Algérie : état des lieux et problématiques. In: Les filières et marchés du lait en méditerranée. Montpellier (France), CIHEAM, Options Mediter., Sér. B, 32 : 25-45

Bene S., Nagy B., Nagy L., Kiss B., Polgar J.P., Szabo F., 2007. Comparison of body measurements of beef cows of different breeds. Arch. Anim. Breed., 50 (4): 363-373, doi: 10.5194/aab-50-363-2007

Birkner J.E., Garcia Vinent J.C., Alberio R.H., Butler H., 1984. The effect of Hereford bull libido on the conception rate of heifers over a 21-day breeding period. Rev. Argentina Prod. Anim. 4 : 1149-1155

Bouzebda-Afri F., Bouzebda Z., Bairi A., Franck M., 2007. Etude des performances bouchères dans la population bovine locale dans l'est algérien. Sci. Technol. C, Biotechnol., 26: 89-97

Chenoweth P.J., Hopkins F.M., Spitzer J.C., Larsen R.E., 2010. Guidelines for using the bull breeding soundness evaluation form. Clin. Theriogenol. 2 (1): 43-50

Couix N., Gaillard C., Lauvie A., Mugnier S., Verrier E. 2016. Des races localement adaptées et adoptées, une condition de la durabilité des activités d'élevage. Cah. Agric., 25 (6): 650009, doi: 10.1051/cagri/2016052

Coulomb J., 1976. N'Dama cattle breed. Some zootechnic characteristics. Rev. Elev. Med. Vet. Pays Trop., 29 (4): 367-380, doi: 10.19182/remvt.8005

Coulter G.H., Foote R.H., 1979. Bovine testicular measurements as indicators of reproductive performance and their relationship to productive traits in cattle: a review. Theriogenology, 11: 297-311, doi: 10.1016/0093$691 X(79) 90072-4$

Coulter G.H., Mapletoft R.J., Kozub G.C., Cates W.F., 1987. Scrotal circumference of two-year-old bulls of several beef breeds. Theriogenology 27: 485-91, doi: 10.1016/0093-691X(87)90236-6

Dasrul D., Wahyuni S., Sugito S., Hamzah A., Zaini Z., Haris A., Gholib G., 2020. Correlation between testosterone concentrations with scrotal circumference, and semen characteristics in Aceh bulls. EDP Sciences, Paris, France, doi: 10.1051/e3sconf/202015101015

Dehoux J.P., Hounsou-Ve G., 1993. Productivité de la race bovine Borgou selon les systèmes d'élevage traditionnels au Nord-Est du Bénin. Rev. Mond. Zootech., 74 (75): 36-48

Delage J., Poly J., Vissac B., 1955. Etude de l'efficacité relative des diverses formules de barymétrie applicables aux bovins. Ann. Zootech., 4 (3): 219 231, doi: 10.1051/animres:19550305

Dumont P., 1997. Appréciation de la fonction sexuelle du taureau reproducteur. Point Vet., 28: 1617-1628

FAO. 2013. Caractérisation phénotypique des ressources génétiques animales. Directives FAO sur la production et la santé animales 11, FAO, Rome, Italie, $151 \mathrm{p}$.

Fordyce G., McGowan M.R., Lisle A., Muller T., Allen J., Duff C., Burns B.M., 2014. Scrotal circumference of Australian beef bulls. Theriogenology, 81 (6): 805-812, doi: 10.1016/j.theriogenology.2013.12.020
Gbangboche A.B., Alkoiret T.I., 2011. Reproduction et production de lait des bovins de race Borgou et N'dama au Bénin. J. Appli. Biosci., 46: 3185-3194

Hanzen C., 2015-2016. In : La propédeutique de l'appareil reproducteur et l'examen du sperme des ruminants. Service de Thériogenologie des animaux de production, Faculté de médecine vétérinaire, Université de Liège

Hartmann W., Pereira J.F.S., Grochoski T.R., Fazzano C.J., Bach R.A. 2018. comparative andrologic evaluation between nellore and brangus bulls. Rev. Eletrôn. Biociênc., Biotecnol. Saúde, 11 (22): 34-42

Hopkins F.M., Spitzer J.C., 1997. The new Society for Theriogenology breeding soundness evaluation system. Vet. Clin North America: Food Anim. Pract. 13 (2): 283-293, doi: 10.1016/S0749-0720(15)30341-8

Kasari T.H., Wikse S.E., Jones R., 1996. Utilisation de taureaux d'un an dans les élevages de bovins de boucherie. Compend. Contin. Educ. Pract. Vét., 18: 1244-1253

Kastelic J.P., Thundathil J.C., 2008. Breeding soundness evaluation and semen analysis for predicting bull fertility. Reprod. Domest. Anim., 43: 368-373, doi : 10.2527/2003.812395x

Marmet R. 1983. La connaissance du bétail : Les bovins. Tome 1. Lavoisier, Cachan, France, 187 p.

McGowan M.R., Bertram J.D., Fordyce G., Fitzpatrick L.A., Miller R.G., Jayawardhana G.A., Doogan V.J., et al., 2002., Bull selection and use in northern Australia. 1. Physical traits. Anim. Reprod. Sci., 71 (1-2): 25-37, doi: 10.1016/S0378-4320 (02)00023-4

Moupandza P., Akouango P., Ngatse S.D., Ngokaka C., 2013. State of the fertility and reproductive efficiency of the herd of Ndama bulls and cows in Abo farms (Congo Brazzaville), J. Appl. Biosci., 64: 4839-4846., doi: 10.4314/jab.v64i1.88473

Orgeur P., Signoret J.P., 1990. L'activité sexuelle du taureau : revue bibliographique. Prod. Anim., 3 (4): 235-242, doi : 10.20870/productions-animales.1990.3.4.4382

Parkinson T.J., 2004. Evaluation of fertility and infertility in natural service bulls. Vet. J. 168: 215-29, doi: 10.1016/j.tvjl.2003.10.017

Petherick J.C., 2005. Animal welfare issues associated with extensive livestock production: The northern Australian beef cattle industry. Appl. Anim. Behav. Sci., 92(3): 211-234, doi: 10.1016/j.applanim.2005.05.009

Rahmani M.M., Hamiroune M., Hammadi M., Fatnassi M., Lamia D., Berber A., 2019. Etude des relations entre circonférence scrotale, testostérone sérique et âge chez des bovins mâles bruns de l'Atlas en Algérie. Livest. Res. Rural Dev., 31: 12

Rahmani M.M., Hamiroune M., Berber A., 2020. Caractérisations morphologique et biométrique de bovins mâles Bruns de l'Atlas en Algérie. Livest. Res. Rural Dev. 32: 2

Schramm R.D., Osborne P.I., Thayne W.V., Wagner W.R., Inskeep E.K., 1989 Phenotypic relationships of scrotal circumference to frame size and body weight in performance-tested bulls. Theriogenology, 31 (3): 495-504, doi: 10.1016/0093-691X(89)90234-3

Soukehal A., 2013. Communications sur la filière laitière. In : Colloque La sécurité alimentaire : quels programmes pour réduire la dépendance en céréales et lait? Alger, Algérie, 8 avr 2013

Yakhlef H., Madani T., Abbache N. 2002. Biodiversité importante pour l'agriculture : cas des races bovines, ovines, caprines et camelines. MATE-GEF/ PNUD, $43 \mathrm{p}$. 


\section{Summary}

Yahimi A., Djellata N., Hanzen C. Some morphometric and reproductive characteristics of Brown Atlas bulls in Algeria

The study describes the morphometric and reproductive parameters, and their influencing factors in 249 Brown Atlas bulls with an average age of $15.3 \pm 3.7$ months and a weight of 165.7 $\pm 50.7 \mathrm{~kg}$. The height at withers, thoracic perimeter and scrotal circumference were $110.8 \pm 8.5 \mathrm{~cm}, 137.2 \pm 13.2 \mathrm{~cm}$ and $25.5 \pm 3.7 \mathrm{~cm}$, respectively. Among the bulls, $12.8 \%$ did not show any libido, in $22.1 \%$ of them it was excellent. The averages of the variables studied according to the age class showed a normal and progressive evolution from one class to another. Although, there were marked variations in the mean values of age (AgeM), height at withers (HGM) and scrotal circumference (CSM) at farm level. Our results also identified highly significant correlations $(p<0.0001)$ between the mean values of chest circumference (TPM), HGM, CSM, and those of weight (PoidsM) and age (AgeM). Similarly, the linear model used showed a very high coefficient of determination $\left(R^{2}\right)$ between TPM, CSM, and AgeM (0.84) and PoidsM (0.62). On the other hand, it was very low $\left(R^{2}=0.35\right)$ between HGM, and AgeM and PoidsM. The analysis of variance of the parameters according to the farm and the age classes showed a highly significant effect $(p<0.0001)$ on the whole population and the 12-18-month class. Regarding libido, a significant effect of scores 2 and 3 for the 12-18-month age class was found.

Keywords: cattle, Brown Atlas bulls, libido, scrotum, Algeria

\section{Resumen}

Yahimi A., Djellata N., Hanzen C. Algunas características morfométricas y de reproducción de los toretes de raza Parda del Atlas en Argelia

El estudio describe los parámetros morfométricos y de reproducción, y los factores influyentes, en 249 toretes de raza Parda del Atlas, con edad promedio de 15,3 $\pm 3,7$ meses y con un peso de $165,7 \pm 50,7 \mathrm{~kg}$. Las alturas a la cruz, los perímetros torácicos y las circunferencias escrotales fueron respectivamente de 110,8 $\pm 8,5 \mathrm{~cm}, 137,2 \pm 13,2 \mathrm{~cm}$ y $25,5 \pm 3,7 \mathrm{~cm}$. En lo que concierne la libido, $12,8 \%$ de los toretes no presentaron ninguna y en $22,1 \%$ de ellos fue excelente. Los promedios de las variables estudiadas en función de la clase de edad presentaron una evolución normal y progresiva de una clase a la otra. Aunque, se constataron variaciones muy netas concernientes a los valores promedios de edad (AgeM), de la altura a la cruz (HGM) y de la circunferencia escrotal (CSM) a nivel de la finca. Nuestros resultados identificaron igualmente correlaciones muy significativas $(p<0,0001)$ entre los valores promedio de la circunferencia del pecho (TPM), de la HGM, de la CSM, y del peso (PoidsM) y de la edad (AgeM). Igualmente, el modelo linear utilizado mostró un coeficiente de determinación (R2) muy fuerte entre TPM, CSM, y AgeM $(0,83)$ y PoidsM $(0,62)$. En cambio, fue muy débil $(R 2=0,35)$ entre HGM, y AgeM y PoidsM. El análisis de varianza de los parámetros en función de la finca y de las clases de edad mostró un efecto muy significativo $(p<0,0001)$ sobre el conjunto del efectivo y de la clase 12-18 meses. Concerniente a la libido, se observó un efecto significativo de puntuaciones 2 y 3 para la clase de edad 12-18 meses.

Palabras clave: ganado bovino, toro Parda del Atlas, líbido, escroto, Argelia 